\title{
Misguidance in diabetes nutrition: Food labeling and agency recommendations
}

\author{
Stephanie Morrison and Jody Schuurman
}

\section{University of Western Ontario}

Walking through the grocery store, perhaps hungry after a workout or a busy day, Canadians are bombarded by snazzy food marketing. "Low-Fat", "High in Fibre", "Zero Trans Fat", "Ancient Grains!", the bright words jump out as we navigate the store trying to make choices that are both good for our bodies and enticing for our appetites. Food labels throughout grocery stores broadcast conflicting and one-sided messages about the health appeal of their respective products: advertising often boldly proclaims the "healthy" aspects of products, while marginalizing those aspects that are less healthy. Packaging for whole-wheat crackers, for example, might boast "high in fibre", while the equally important health-related reality of the crackers' high sodium content is only subtly revealed in the requisite Nutritional Information fine print. For consumers, choosing foods that will fuel us appropriately and keep us healthy is not a new problem, but the variety of food products becoming available to us, and their prolific marketing is an overwhelming factor in the diabetes epidemic in Canada. ${ }^{1}$

Research has indicated that it is easy to be swayed by the claims made on food packages. ${ }^{2}$ The tendency of food advertising to overemphasize the nutritional quality and health benefits of certain products tends to misinform consumers, which can create barriers to making healthy food choices. For instance, overemphasizing and over advertising positive health-related qualities of foods purposefully omits less positive realities. One example of this selective advertising can be found among certain yoghurt products, which often boast their excellent calcium content and avoid revealing their particularly high sugar content.

Eating well in spite of selective food advertising becomes even more complicated for individuals living with diabetes. In order to manage their health, people living with diabetes are often implored to use complicated paradigms that include attuning to glycemic loading, tracking fasting blood glucose levels, and striving to reach glycated hemoglobin targets. ${ }^{3}$ These strategies are typically an adjunct to the already challenging practice of calorie counting. Navigating these complicated management paradigms is exacerbated by targeted advertising on food packages.

Nutrition management of diabetes is further being derailed with ambiguous and often incongruent messages about healthy eating from public health entities. For example, although the Canadian Diabetes Association (CDA) does its best to simplify healthy eating in its "Just the Basics" publication, which is part of the clinical practice guidelines for healthcare professionals, ${ }^{4}$ their information can be abstruse. A sample meal plan describes two options, one for small appetites (approximately 1800 calories) and another for large appetites (approximately 3200 calories), with a startling caloric difference between the two menus. A subjective description of "small" and "large" appetites, without further explanation or even a calorie count to accompany the meal plan creates ambiguity and confusion.

Another discrepancy in the CDA's "Just the Basics" publication lies in its breakfast suggestions, which include cold cereal as a healthy option, despite the presence of large amounts of sugar in many cereal products. ${ }^{5}$ The CDA best practice guidelines emphasize the importance of paying attention to the glycemic index of foods for optimal diabetes nutrition, but then their choice to list cereal as a 'healthy' breakfast option ignores the probability of glycemic spiking that comes along with the high sugar content in many commercially available cereals. In this sense, the CDA's guidelines for diabetes management are not in synch with the CDA's own food intake recommendations.

In an effort to make food choices easier, the Heart and Stroke Foundation created "Health Check", a labelling system designed to help Canadians eat well. ${ }^{6}$ This is a great idea in theory, but it is facing increasing criticism for giving 
healthy ratings to products high in sodium and therefore not conducive to healthy eating. ${ }^{7}$ One of the challenges with the "Health Check" approach is that nutrition experts are not unified in their opinions about what constitutes healthy eating. A variety of recommendations such as the DASH (Dietary Approaches to Stop Hypertension) diet, the Glycemic Load eating strategy, the Mediterranean diet, and low-carbohydrate diets have all been touted at different points as the "best diet" for people living with diabetes. Interestingly, a recent randomized controlled trial found that people eating different macronutrient ratios experienced similar weight loss, ${ }^{8}$ a common goal for people managing Type II diabetes. These weight-loss results are consistent with reviews of various diets for people living with diabetes. ${ }^{9}$ Many of these "best diets" have underlying common traits, such as eating a wide variety of fruits and vegetables, eating foods that are good sources of fibre, and reducing intake of trans and saturated fats. Taken together, it appears the underlying answer to healthy eating for prevention and management of diabetes may ultimately be simplified through quality food choices and portion control. ${ }^{9}$

We suggest that a public health approach to reducing the current diabetes epidemic should involve improving the eating patterns of all Canadians by empowering consumers to make good choices. We believe that Canadians want to eat a healthy diet, but efforts to do so have been hampered by the bombardment of health-claims in food marketing and by the dissemination of confusing messages about healthy eating, including, some from well-intentioned public health agencies. We encourage Canadian regulators to consider the broad effects of marketing pre-packaged food as 'healthy' and we hope that agencies such as the CDA and the Heart and Stroke Foundation will streamline messages and recommendations for healthy eating.

\section{References}

1. Tarasuk V. Policy directions to promote healthy dietary patterns in Canada. Appl Physiol Nutr Metab. 2010 Apr;35(2):229-233.

2. Labiner-Wolfe J, Jordan Lin CT, Verrill L. Effect of low-carbohydrate claims on consumer perceptions about food products' healthfulness and helpfulness for weight management. J Nut Educ Behav. 2010 Sept;42(5):315-20.

3. Canadian Diabetes Association Clinical Practice Guidelines Expert Committee. CanadianDiabetes Association 2008 clinical practice guidelines for the prevention and management of diabetes in Canada. Can J Diabetes. 2008; 32(suppl 1):S1-201.

4. Canadian Diabetes Association. Just the Basics, Health Eating for Diabetes Management and Prevention. Toronto(ON): Canadian Diabetes Association; 2010 Mar. 4 Report No. 111015 08-365 03/10 Q-400M.

5. Pestano P, Yeshua E, Houlihan J. Sugar in children's cereals: Popular brands pack more sugar than snack cakes and cookies. Washington (DC): Environmental Working Group; 2011 Dec. 23.

6. Heart \& Stroke Health CheckTM. Heart and Stroke Foundation [Internet] 2011 [cited 2012 Feb 1]. Available from: http://www.healthcheck.org/ page/what-health-check

7. Freedhoff, Y. Health Check Program. Can Med Assoc J. 2008 April;178(9):1188.

8. de Souza RJ, Bray GA, Carey VJ, Hall KD, LeBoff MS, Loria CM, Laranjo NM, Sacks FM, Smith SR. Effects of 4 weight-loss diets differing in fat, protein, and carbohydrate on fat mass, lean mass, visceral adipose tissue, and hepatic fat: results from the POUNDS LOST trial. Am J Clin Nutr. 2012 Jan; Epub ahead of print. Available from PubMed: www.ncbi.nlm.nih.gov/ pubmed/22258266

9. Magkos F, Yannakoulia M, Chan JL, Mantzoros CS. Management of the metabolic syndrome and type II diabetes through lifestyle modification. Annu Rev Nutr. 2009 Aug;29:223-256.

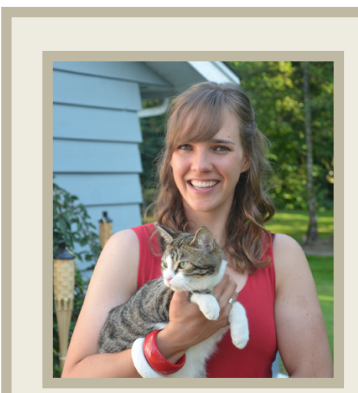

\section{Jody Christine Schuurman}

Jody is a Masters candidate in Kinesiology and a varsity rower at the University of Western Ontario. She is currently studying intensive lifestyle interventions using remote monitoring for people with type 2 diabetes under Dr. Robert Petrella. Her passions are promoting active living, healthy eating and integrating theory and research with current technology to help people make and sustain the behaviour changes they want to achieve.

\section{Stephanie J. Morrison}

Stephanie is a Masters candidate in Health and Rehabilitation Sciences at University of Western Ontario where she investigates strategies for improving gait patterns in individuals with Parkinson's disease. Her apparently divergent interests in Parkinson's and diabetes are firmly grounded in her passion for promoting optimized functioning and health for all individuals.

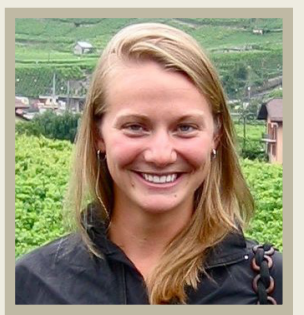

